\title{
Nanoscale Mechanical Stimulation Method for Quantifying C. elegans Mechanosensory Behavior and Memory
}

\author{
Takuma SugI, ${ }^{* 1, * 2, * 3 \dagger}$ Etsuko OKUMURA, ${ }^{* 3, * 4}$ Kaori KISO, ${ }^{* 3}$ and Ryuji IgARASHI*1,*5 \\ *1 PRESTO, Japanese Science and Technology Agency, 4-1-8 Honcho, Kawaguchi, Saitama 332-0012, Japan \\ *2 Molecular Neuroscience Research Center, Shiga University of Medical Science, Otsu, Shiga 520-2192, Japan \\ *3 Institute for Integrated Cell-Material Sciences (WPI-iCeMS), Kyoto University, Yoshida-Honmachi, Sakyo, \\ Kyoto 606-8501, Japan \\ *4 Laboratory of Terrestrial Microbial Ecology, Graduate School of Agriculture, Kyoto University, \\ Kitashirakawa-Oiwake-cho, Sakyo, Kyoto 606-8502, Japan \\ *5 Department of Molecular Engineering, Graduate School of Engineering, Kyoto University, Nishikyo, \\ Kyoto 615-8510, Japan
}

\begin{abstract}
Withdrawal escape response of C. elegans to nonlocalized vibration is a useful behavioral paradigm to examine mechanisms underlying mechanosensory behavior and its memory-dependent change. However, there are very few methods for investigating the degree of vibration frequency, amplitude and duration needed to induce behavior and memory. Here, we establish a new system to quantify C. elegans mechanosensory behavior and memory using a piezoelectric sheet speaker. In the system, we can flexibly change the vibration properties at a nanoscale displacement level and quantify behavioral responses under each vibration property. This system is an economic setup and easily replicated in other laboratories. By using the system, we clearly detected withdrawal escape responses and confirmed habituation memory. This system will facilitate the understanding of physiological aspects of C. elegans mechanosensory behavior in the future.
\end{abstract}

Keywords Mechanosensory behavior, memory, withdrawal escape response, C. elegans

(Received April 12, 2016; Accepted April 26, 2016; Published November 10, 2016)

\section{Introduction}

Mechanical forces such as touch, vibration and gravity make vital influences on the development and homeostasis of living organisms. ${ }^{1,2}$ Animals cope with these stimuli by modifying behavior and thereby achieve physical interactions with the environment. ${ }^{3}$ The mechanism underlying mechanosensory behavior has been studied on various model organisms from bacteria and archaea to mammals. ${ }^{4,5}$ However, physiological aspects of those behaviors, such as force and duration enough to sense, are not well understood.

Mechanosensory behavior in C. elegans is a traditional paradigm in which to examine the mechanism underlying a response to mechanical forces. ${ }^{6-8}$ Worms usually exhibit a withdrawal escape response to anterior body touch and nonlocalized vibrations such as the tapping of a cultivated Petri plate. $^{7}$ The nonlocalized vibration simultaneously stimulates the anterior and posterior touch receptor neurons, and the predominate response for this combined stimulation is reversal movement in naive worms. ${ }^{9}$ After spaced training for repeated mechanical tap stimulation, trained worms can habituate to the stimulus and exhibit a decrease in the magnitude of the

$\dagger$ To whom correspondence should be addressed.

E-mail: tsugi@belle.shiga-med.ac.jp withdrawal escape response. Therefore, worms can alter behavior based on their past experience. ${ }^{10}$ Because the neural circuit underlying this behavior was completely determined, we can easily investigate neural and molecular mechanisms using C. elegans cell-specific genetic methods. ${ }^{10-12}$

In general, the choice of technique to impose mechanical stimulation and to readout behavioral output is critical for understanding the physiology of animal behavior. In C. elegans, nonlocalized mechanical stimulation has been provided by tapping only a single Petri plate using a solenoid tapper ${ }^{13}$ or a ROBO cylinder, ${ }^{12}$ by manually dropping a plastic box containing multiple plates from a constant height ${ }^{10}$ or by playing sound to evoke vibration of an agar surface of a plate using dual cone speaker. ${ }^{14}$ However, there have been very few methods that allow for precisely changing the vibration properties such as frequency, amplitude and duration while also validating these properties quantitatively.

At a cellular level, several mechanical stimulation methods have been also established using mammalian cells for the study of mechanotransduction. ${ }^{15}$ These methods include mechanoclamp using a piezo-driven system, ${ }^{16,17}$ surface elongation of a flexible silicone elastomer, ${ }^{18,19}$ and force application by magnetic particles..$^{20-22}$ In addition to these spatially confined methods, Nikukar et al. recently reported an interesting method, "nanokicking", using a piezo actuator and it was demonstrated to evoke nanoscale nonlocalized vibration with high frequency 

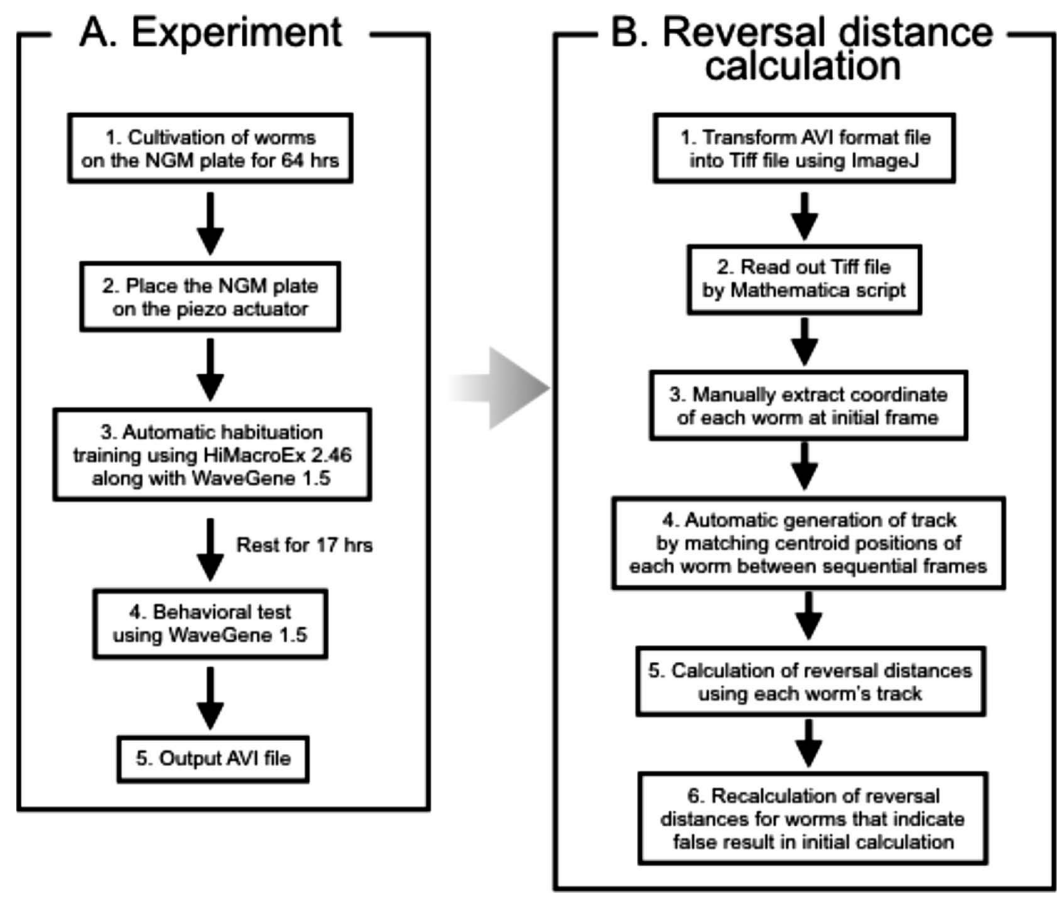

Fig. 1 Flowchart for quantification of C. elegans mechanosensory behavior and memory. (A) Experimental procedure. The flowchart is indicated for examining mechanosensory memory. In the case of only examining behavioral response to mechanical stimulus, step 3 could be skipped. (B) Calculation procedure. The detailed instructions are described in a supplementary file.

(up to $1 \mathrm{kHz}$ ) across the entire surface of the Petri plates. ${ }^{23,24}$ However, this strategy has not been applied to a living animal level experiment, particularly to its behavioral experiments.

In this study, we designed an economic nonlocalized vibration device using a piezoelectric sheet speaker. This device allows for elaborately changing the vibration parameters and setting these parameters in a desired temporal pattern. We also quantitatively validated the vibration properties using laser vibrometer. Using our new device, we clearly quantified the reversal responses and memory of worms for nonlocalized vibration. Thus, our new device will facilitate understanding of the physiological aspects of C. elegans mechanosensory behavior by titrating vibration properties in the future.

\section{Experimental}

\section{Strain preparation}

We used wild-type N2 Bristol strain for all behavioral experiments. This strain has been maintained and handled using standard methods. ${ }^{25}$

\section{Vibration device construction and its validation}

To control properties of nonlocalized vibration precisely, a new system was constructed using the piezoelectric sheet speaker (THRIVE, pzBAZZ $\mu$ Speaker B35) as an actuator and the amplifier module (THRIVE, 0530AMPZ) connected to a computer earphone jack via an earphone splitter. The diameter of the sheet speaker was $42 \mathrm{~mm}$, and the frequency could be increased at least up to $5 \mathrm{kHz}$. The amplitude could be also changed by the volume control of the computer in the range of 0 to $100 \%$ (Dell T1650 desktop computer). The minimum duration of vibration is $0.1 \mathrm{~s}$. The mechanical stimuli were quantified by laser vibrometer V100 (Denshigiken Corp.) with a PicoScope oscilloscope.

\section{Behavioral recording}

All worms' behaviors were recorded at $>7.0$ frames/s using USB-controlled CCD cameras (Sentech, STCTB83USB-AS), which were each coupled to a $25 \mathrm{~mm}$ focal-length $\mathrm{C}$-mount machine vision lens (Azure, AZURE-2514MM) and C-mount adaptor (5 mm thickness, 30CMA-R). Each pixel in the captured images corresponds to a $25.4 \times 25.4 \mu \mathrm{m}$ area in each Petri plate. The total field captured was $26.0 \times 19.5 \mathrm{~mm}$.

\section{Software}

Two free download pieces of software were used for automatic stimulation; WaveGene Ver. 1.50 for control of vibration properties and mouse macro HiMacroEX 2.46 for automatic habituation instead of manual operation. The software was compiled and bench marked on a Dell PRECISION T1650 desktop computer (Dell). The script written in HiMacroEx 2.46 is indicated in Fig. 3.

The reversal distance was calculated according to a previously reported method. ${ }^{12}$ At first, an acquired AVI format movie was transformed into Tiff-format sequential images using ImageJ software $(\mathrm{NIH})$. This sequential image file was used for subsequent motion analysis of each worm. Image-processing software (Reversal Calculator Ver. 1, Supporting Information) for the quantification of each worm's reversal distance was written in Mathematica 9.0 (Wolfram). The coordinate of each worm at the initial frame was extracted manually. Then, the centroid of each worm was calculated and a track was generated by matching centroid positions between sequential frames. This track was used to calculate the reversal distance. Initially, the reversal distance of each worm was calculated automatically by the software. After this initial calculation, a frame number in which the reversal movement was completed was manually confirmed for each worm using the output result. Then, correct frame numbers for worms that indicate incorrect frame numbers in the initial calculation were extracted manually and put into 

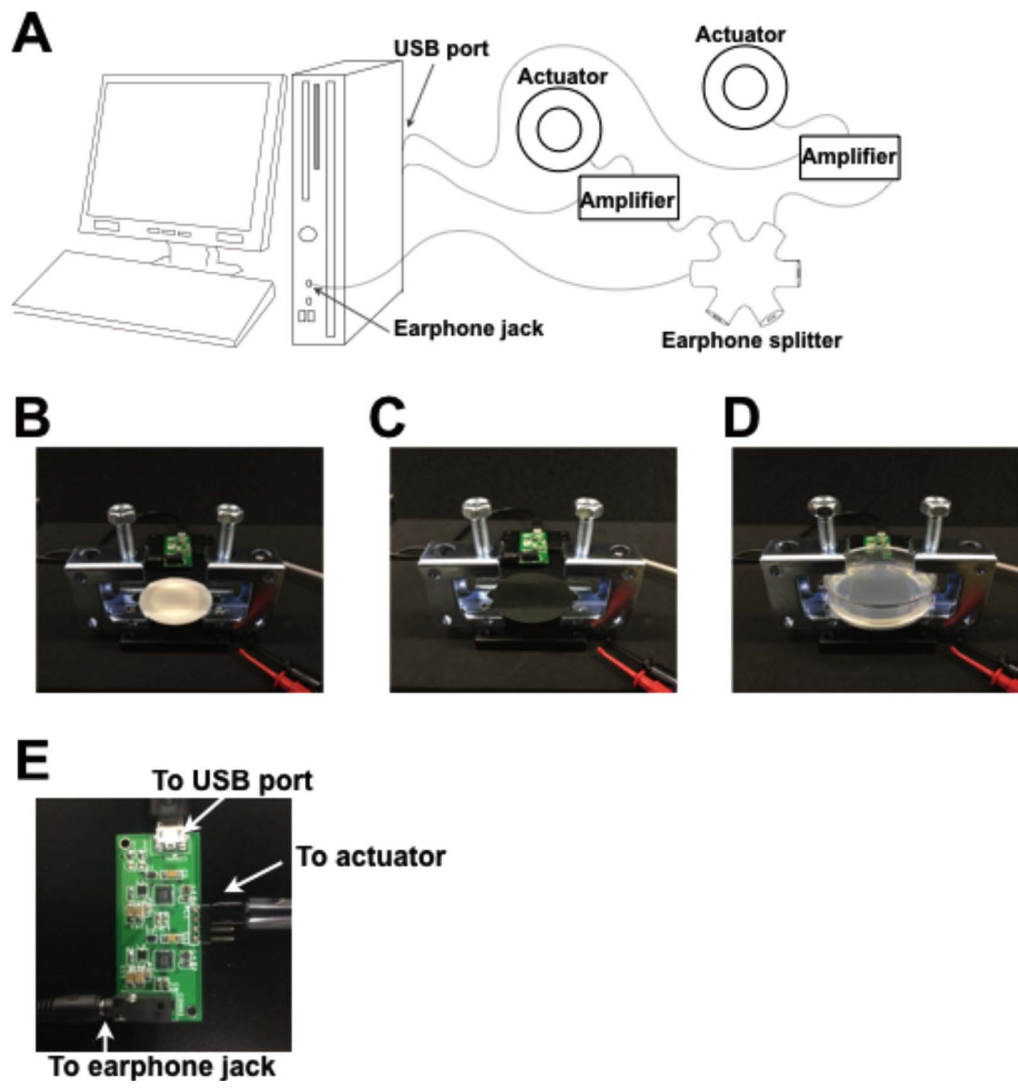

Fig. 2 Design of a new nonlocalized stimulation device. (A) Schematic overview of the new device. (B - D) Photos of an actuator without (B) and with a black sheet (C), and with both the black sheet and a Petri plate (D). A black sheet is required for enhancing the contrast between worms and background in each acquired image. The Petri plate was placed on the piezoelectric sheet speaker, and only the two peripheral positions of the Petri plate and speaker were fixed on the stage using the two screws. (E) Amplifier connected to an actuator and a computer.

the software for recalculation of their reversal distances. The detailed instructions for this software are described in a supplemental file. The software is available on request.

\section{Habituation training}

Worms were cultivated on $60 \mathrm{~mm}$ Petri plates (Thermo Fisher Scientific, \#150288) containing $10 \mathrm{~mL}$ of NGM with $2 \%$ agar, on which Escherichia coli OP50 was seeded. On the first day, 8 - 9 worms were deposited onto each NGM plate and cultivated at $20^{\circ} \mathrm{C}$. After $3 \mathrm{~h}$, the deposited $\mathrm{P} 0$ worms were removed to segregate the F1 progeny. The progeny were cultivated for $64 \mathrm{~h}$ at $20^{\circ} \mathrm{C}$. An NGM plate on which worms were cultivated was placed on an actuator of a piezoelectric sheet speaker and stimulated through WaveGene. The training protocol was flexibly customized using mouse macro HiMacroEX 2.46. In this study, the conventional training ${ }^{26}$ was adopted for worms within a $20^{\circ} \mathrm{C}$ incubator (Advantec). The nonlocalized vibrations were evoked at every $1 \mathrm{~min}$ for 20 times, and this stimulation sequence was repeated five times with a $1 \mathrm{~h}$ interval.

\section{Results and Discussion}

Device for evoking mechanical stimuli

We previously established the tap stimulation system using the cylinder and actuator, because this method was successfully applied to the quantification of tap habituation behavior in other groups. $^{12,13}$ In this system, amplitude of the nonlocalized vibration could be roughly changed, whereas its frequency and duration could not. Therefore, instead of the previous system, we used a piezoelectric speaker to evoke nonlocalized vibration on an agar surface of an NGM plate (Figs. 1 and 2A). The NGM plate on which worms were cultivated (Fig. 1A Step 1) was placed on a circular-shaped actuator of a piezoelectric speaker (Fig. 1A Step 2, and Figs. 2B - 2D). The actuator was connected to an amplifier (Fig. 2E). This device was also connected to an earphone jack of a desktop computer through an earphone splitter. The earphone splitter enabled us to evoke nonlocalized vibration to multiple NGM plates at the same time.

Amplitude of vibration can be set by the volume control of a computer, and this sound volume level was changed in the range of 0 to $100 \%$. The free download software was used for setting the frequency and duration of the vibration. The frequency of vibration can be changed in the range of 0 to $5 \mathrm{kHz}$. The minimum duration of stimulation is $0.1 \mathrm{~s}$. Moreover, this devise potentially enables us to generate various waveforms such as sine wave, square wave, pulse wave and white noise.

Furthermore, semi-automation of the training of the worms was also needed for quantifying habituation memory due to its laborious protocol. The conventional training protocol consisted of five blocks of 20 mechanical stimuli $(60 \mathrm{~s}$ interstimulus interval) with a $1 \mathrm{~h}$ rest period between each block. ${ }^{26}$ To automatically train large populations of worms, we used a mouse macro system that enabled us to program the automatic 


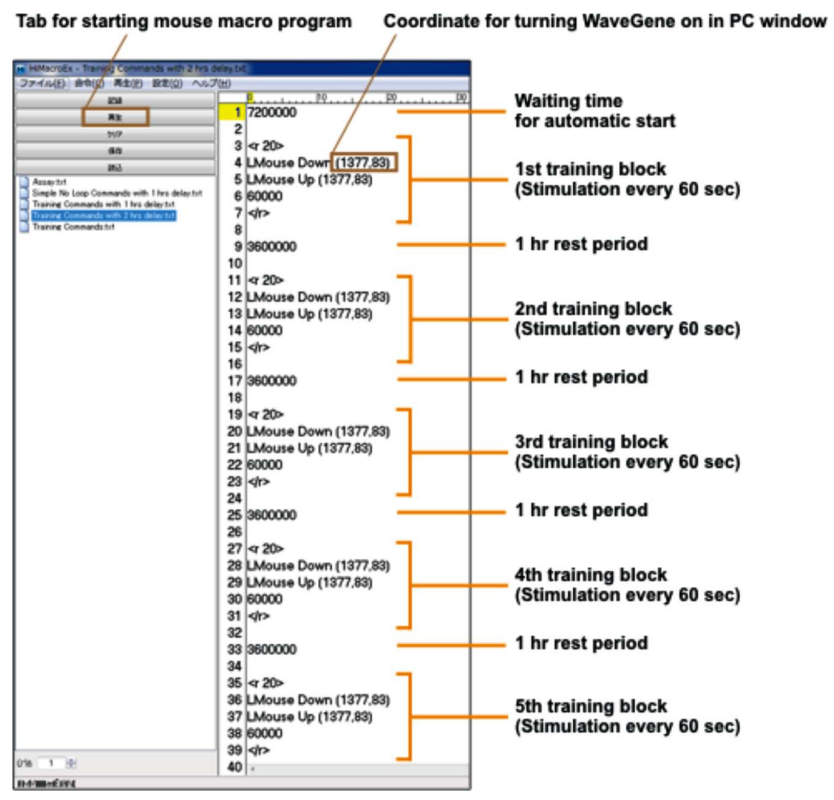

Fig. 3 Script written in mouse macro system HiMacroEX 2.46. The script for running conventional training protocol (five blocks of 20 tap stimuli (60 s interstimulus interval) with a $1 \mathrm{~h}$ rest period between each block) was described in the mouse macro system. Coordinate for turning WaveGene on was changed on the basis of the position of the WaveGene window. Description of waiting time at the beginning of the script enables automatic start of the mouse macro program.

mouse cursor movement on the computer screen (Fig. 1A Step 3 and Fig. 3). To accurately examine habituation memory of trained worms, we simultaneously prepared untrained worms as a control.

\section{Quantification of mechanical stimuli evoked by a new system}

We used laser interferometry to quantify mechanical stimuli evoked by our piezoelectric speaker system. We played a $440 \mathrm{~Hz}$ sound (the standard tuning frequency) on a piezoelectric speaker and validated the vibration on the center of an agar surface in a Petri plate (Fig. 4A). Expectedly, as shown in Fig. 4B, we succeeded in detecting a $440 \mathrm{~Hz}$ frequency. Therefore, we have changed the vibration frequency on the center of agar surfaces using WaveGene (Fig. 4C). All the vibration frequencies determined by WaveGene (minimum frequency, $250 \mathrm{~Hz}$ ) were clearly detected by the vibrometer. In addition, an $80 \mathrm{~Hz}$ vibration was correctly detected by the accelerometer. These results indicate that our piezoelectric speaker system allows for evoking vibration with accurate frequency on the center of an agar surface.

We have changed the amplitude of vibration by volume control of the computer. As shown in Fig. 4D, the amplitude quantified by the vibrometer on an agar surface linearly increased as the sound volume level increased, and reached a plateau at $80 \%$ of the computer sound level. Importantly, we could detect $1 \mathrm{kHz}$ vibrations with 10 and $20 \%$ of the computer sound level as the nanometer scale displacements. Therefore, we next changed the sound level in the range of 0 to $30 \%$ by $2 \%$ and quantified the displacement in each sound level (Fig. 4E). We could detect the $30.4 \mathrm{~nm}$ displacement in $2 \%$ of the sound level, and the displacement increased within a nanometer range as the sound volume level became higher. These results reveal that our economic piezoelectric speaker system allows for changing amplitude of the vibration at a nanoscale resolution.
Then, we tried to measure $1 \mathrm{kHz}$ vibration (4\% of the computer sound level) evoked by WaveGene at several positions on the agar surface (Figs. 4A and 4F). As a result, we detected almost no frequency deviations across the surface of the Petri plate. These results indicate that our piezoelectric speaker enables us to stimulate almost all the worms under the same vibration properties, regardless of their positions on an agar surface.

\section{Proof of principle experiment}

We prepared both untrained and trained worms and quantified their mean reversal distances at $16 \mathrm{~h}$ after habituation training (Fig. 1A Steps 4 - 5, Figs. 1B Steps 3 and 5). Untrained worms exhibited reversal responses to vibration with $1 \mathrm{kHz}$ of frequency, $4.9 \mu \mathrm{m}$ of amplitude (computer sound level, 100\%) and $1 \mathrm{~s}$ of duration (Figs. $5 \mathrm{~A}$ and $5 \mathrm{C})$. The mean $( \pm \mathrm{SEM})$ reversal distance of these untrained worms $(N=110)$ in response to vibration was $1.82 \mathrm{~mm}( \pm 0.10 \mathrm{~mm})$ (Fig. $5 \mathrm{C})$. On the other hand, in our previous tapping method, the mean reversal distance of worms $(N=98)$ was $1.07 \mathrm{~mm}( \pm 0.06 \mathrm{~mm})$ (Fig. $5 \mathrm{C})$, which was comparable with that reported in the other group's paper. ${ }^{13}$ Therefore, these quantifications have indicated that worms stimulated by our new system could exhibit longer reversal distances than those stimulated by the old tapping system.

We further examined habituation memory of trained worms (Fig. 5B). The mean reversal distance of the trained worms $(N=135)$ in response to vibration was $1.36 \mathrm{~mm}( \pm 0.09 \mathrm{~mm})$. This result revealed that worms trained by our new system showed significantly reduced reversal distance compared with untrained worms (Fig. 5C). Thus, our new system could clearly induce mechanosensory memory through habituation training.

In summary, our system allows for not only quantification of mechanosensory behavior but also training of worms and quantifications of their memory. One of the important advantages for the researcher is that the new system is an economic setup (< approximately 130 dollars/a single vibration device) and easily replicated in other laboratories. In addition, we can flexibly change the vibration properties, and our behavioral experiments have proved the capability of this system. Furthermore, this system potentially promises its throughput and versatility. Besides the inherent throughput of the nonlocalized stimulation methods that allow for simultaneously imposing vibration on large numbers of worms, increase of actuators enables further high-throughput experiments. In terms of versatility, this system is so compact as to be integrated into another experimental device such as a calcium imaging system.

Recently, Goodman's and Pruitt's groups have established a microelectromechanical system (MEMS)-based force clamp system that can noninvasively apply calibrated forces to freely moving worms. ${ }^{27,28}$ This system made it possible to quantitatively estimate force and indentation depths needed for touch-evoked withdrawal response. The response evoked by localized touch stimulation is the result of activation of either anterior or posterior touch receptor neurons. ${ }^{29}$ On the other hands, the reversal responses evoked by tap and our new methods are thought to be the net effect of simultaneously stimulating those neurons. ${ }^{9}$ Therefore, by comparing physiological data obtained from localized and nonlocalized stimulation experiments, we will understand neural cording of vibration properties needed to evoke mechanosensory behavior in the future. 

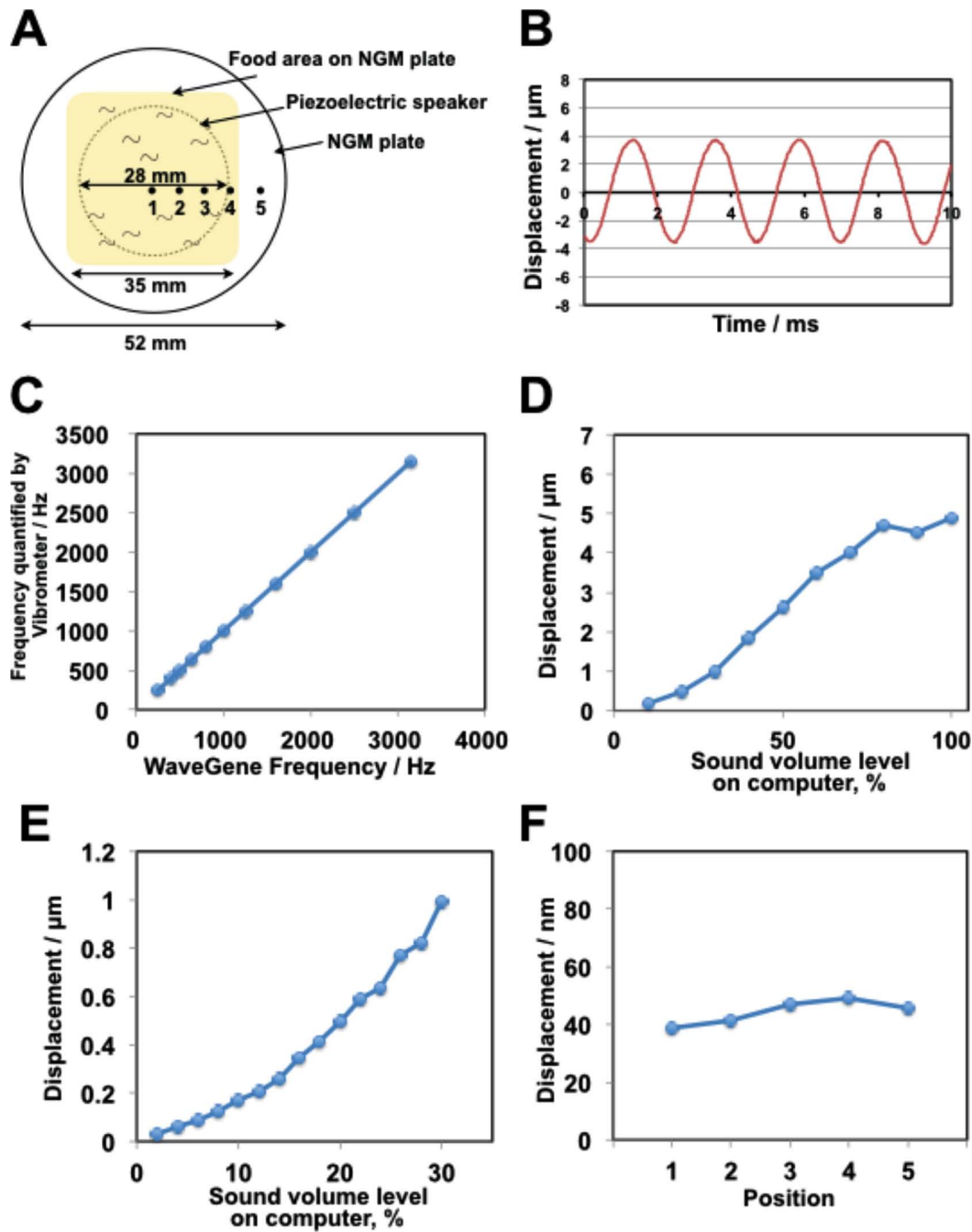

Fig. 4 Validation of the new device by laser interferometry. (A) Schematic of the Petri plate, the piezoelectric speaker, and food area on the agar surface. The speaker is represented by the dashed line. Displacement was quantified at the indicated positions (1 to 5) as described in Fig. 4F. (B) Detection of the standard tuning frequency $(440 \mathrm{~Hz})$ by laser interferometry. The laser was focused on the center of an agar surface. (C) Quantification of various frequencies of vibrations evoked by the new device. The frequency of the evoked vibration was changed using WaveGene, and quantifications were performed by laser interferometry. The computer sound level was set as 50\%. (D) Quantifications of the displacements induced by $1 \mathrm{kHz}$ vibration in the sound level range of 0 to $100 \%$. (E) Detailed quantifications of the displacements induced by $1 \mathrm{kHz}$ vibration, in which the computer sound level was changed in the range of 0 to $30 \%$ by $2 \%$. (F) Quantifications of the displacements at several positions on the agar surface. A vibration of $1 \mathrm{kHz}$ (computer sound level, 4\%) was evoked by WaveGene. Numbers represent distances from the center (1, center; 2, $5 \mathrm{~mm}$ from center; 3, $10 \mathrm{~mm}$ from center; 4, $15 \mathrm{~mm}$ from center; $5,20 \mathrm{~mm}$ from center).

\section{Acknowledgements}

We thank Caenorhabditis Genetic Center for sharing strains. T. S. and R. I. were supported by the Japan Society for the Promotion of Science, Japan Science and Technology Agency under Precursory Research for Embryonic Science and Technology (PRESTO). T. S. was supported by the Mochida Memorial Foundation for Medical and Pharmaceutical Research.

\section{Supporting Information}

This material is available free of charge on the Web at http:// www.jsac.or.jp/analsci/.

\section{References}

1. B. D. Hoffman, C. Grashoff, and M. A. Schwartz, Nature, 2011, 475, 316.

2. P. Delmas, J. Hao, and L. Rodat-Despoix, Nat. Rev. Neurosci., 2011, 12, 139.

3. P. Ramdya, P. Lichocki, S. Cruchet, L. Frisch, W. Tse, D. Floreano, and R. Benton, Nature, 2015, 519, 233.

4. O. P. Hamill and B. Martinac, Physiol. Rev., 2001, 81, 685.

5. T. Iskratsch, H. Wolfenson, and M. P. Sheetz, Nat. Rev. Mol. Cell Biol., 2014, 15, 825.

6. M. Chalfie, Nat. Rev. Mol. Cell Biol., 2009, 10, 44.

7. T. Bozorgmehr, E. L. Ardiel, A. H. McEwan, and C. H. Rankin, Front Physiol., 2013, 4, 88. 
A

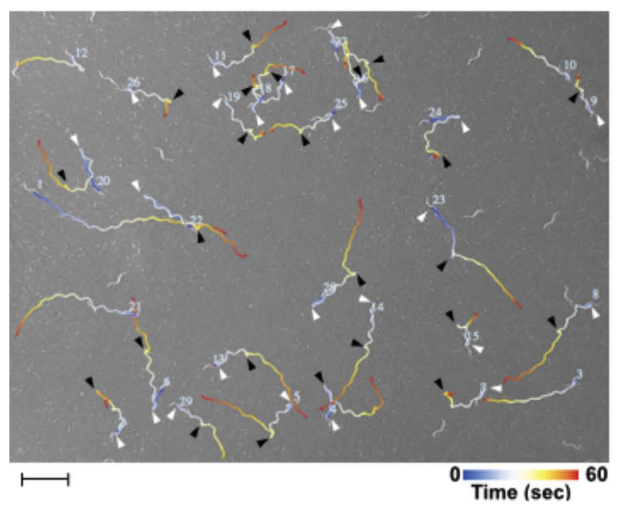

B

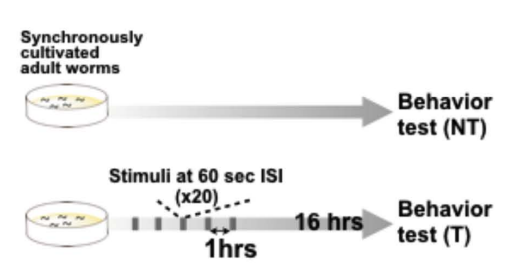

C

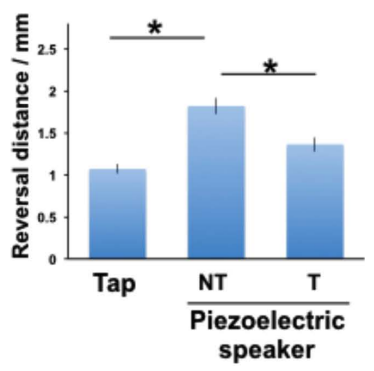

Fig. 5 Behavioral experiments. (A) Trajectories of worms' reversal responses to nonlocalized vibration induced by the new system. The vibration with $630 \mathrm{~Hz}$ of frequency, $4.5 \mu \mathrm{m}$ of amplitude (computer sound level, 50\%), $1 \mathrm{~s}$ of duration was delivered to the Petri plate at $10 \mathrm{~s}$ after starting behavioral recording. The white and black arrow heads indicate start and end positions of each worm's reversal movement, respectively. Worms that suddenly accelerated in a forward movement in response to vibration were not marked by the arrow heads. Scale bar, $2 \mathrm{~mm}$. (B) Scheme of behavioral experiments. The conventional protocol (five blocks of 20 tap stimuli $(60 \mathrm{~s}$ interstimulus interval) with a $1 \mathrm{~h}$ rest period between each block) was used for habituation training. Habituation training was conducted inside an incubator and simultaneously applied for the five plates. Behavioral quantifications were performed at $16 \mathrm{~h}$ after habituation training. (C) Quantifications of mechanosensory behavior and memory using the old tap system and the new piezoelectric sheet speaker system. Reversal distances of worms that were not trained (NT) and trained with the conventional protocol (T) were quantified at $16 \mathrm{~h}$ after training. The vibration with $1 \mathrm{kHz}$ of frequency, $4.9 \mu \mathrm{m}$ of amplitude (computer sound level, $100 \%), 1 \mathrm{~s}$ of duration was delivered to the Petri plate at $10 \mathrm{~s}$ after starting behavioral recording. More than 80 worms were examined in each experimental condition. Error bars indicate SEMs. Statistical comparisons were performed using $t$ tests. $* P<0.01$.

8. P. Delmas and B. Coste, Cell, 2013, 155, 278.

9. S. R. Wicks and C. H. Rankin, J. Comp. Physiol. A, 1996, 179,675 .

10. T. A. Timbers and C. H. Rankin, Behav. Neurosci., 2011, 125,560 .

11. M. Chalfie, J. E. Sulston, J. G. White, E. Southgate, J. N. Thomson, and S. Brenner, J. Neurosci., 1985, 5, 956.

12. T. Sugi, Y. Ohtani, Y. Kumiya, R. Igarashi, and M. Shirakawa, Proceedings of the National Academy of Sciences, 2014, 111, 17236.

13. N. A. Swierczek, A. C. Giles, C. H. Rankin, and R. A. Kerr, Nat. Meth., 2011, 8, 592.

14. X. Chen and M. Chalfie, J. Neurosci., 2014, 34, 6522.

15. J. Hao and P. Delmas, Nat. Protocol., 2011, 6, 979.

16. G. C. McCarter, D. B. Reichling, and J. D. Levine, Neurosci. Lett., 1999, 273, 179.

17. B. Coste, J. Mathur, M. Schmidt, T. J. Earley, S. Ranade, M. J. Petrus, A. E. Dubin, and A. Patapoutian, Science, 2010, 330, 55.

18. C.-M. Cheng, Y.-W. Lin, R. M. Bellin, R. L. Steward, Y.-R. Cheng, P. R. LeDuc, and C.-C. Chen, Nat. Protocol., 2010, 5,714 .

19. M. R. C. Bhattacharya, D. M. Bautista, K. Wu, H. Haeberle,
E. A. Lumpkin, and D. Julius, Proceedings of the National Academy of Sciences, 2008, 105, 20015.

20. P. Tseng, J. W. Judy, and D. Di Carlo, Nat. Meth., 2012, 9 , 1113.

21. F. Etoc, D. Lisse, Y. Bellaiche, J. Piehler, M. Coppey, and M. Dahan, Nat. Nanotechnol., 2013, 8, 193.

22. N. J. Sniadecki, Endocrinology, 2010, 151, 451.

23. H. Nikukar, S. Reid, P. M. Tsimbouri, M. O. Riehle, A. S. G. Curtis, and M. J. Dalby, ACS Nano, 2013, 7, 2758.

24. P. G. Childs, C. A. Boyle, G. D. Pemberton, H. Nikukar, A. S. G. Curtis, F. L. Henriquez, M. J. Dalby, and S. Reid, Acta Biomater, 2016, 34, 159.

25. S. Brenner, Genetics, 1974, 77, 71.

26. J. K. Rose, K. R. Kaun, and C. H. Rankin, Learn Mem., 2002, 9, 130.

27. B. C. Petzold, S.-J. Park, E. A. Mazzochette, M. B. Goodman, and B. L. Pruitt, Integr. Biol. (Camb), 2013, 5, 853.

28. A. L. Eastwood, A. Sanzeni, B. C. Petzold, S.-J. Park, M. Vergassola, B. L. Pruitt, and M. B. Goodman, Proceedings of the National Academy of Sciences, 2015, 112, E6955.

29. S. R. Wicks and C. H. Rankin, J. Neurosci., 1995, 15, 2434. 